The Arithmetic of Discounting

\author{
Peter R. Killeen
}

Arizona State University

Killeen, P. R. (2015). The arithmetic of discounting. Journal of the Experimental Analysis of Behavior, 103, 1, 249-259, DOI: 10.1002/jeab.130

killeen@asu.edu 


\begin{abstract}
Most current models of delay discounting multiply the nominal value of a good whose receipt is delayed, by a discount factor that is some function of that delay. This article reviews the logic of a theory that discounts the utility of delayed goods by adding the utility of the good to the disutility of the delay. In limiting cases it approaches other familiar models, such as hyperbolic discounting. In non-limit cases it makes different predictions, generally requiring, inter alia, a magnitude effect when the value of goods is varied. A different theory is proposed for conditioning experiments. In it utility is computed as the average reinforcing strength of the stimuli that signal the delay. Both theories are extended to experiments in which degree of preference is measured, rather than adjustment to iso-utility values.
\end{abstract}

Keywords: additive utilities, adjusting procedure, delay discounting, magnitude effect, preference procedure, Thurstone scaling 


\section{The arithmetic of discounting}

All popular models of delay discounting assume that a proper covering model computes the current value of a delayed good by multiplying the amount-to-be discounted by some fraction that is a function of time: $v_{\text {Disc }}=v_{\text {Nom }} f(d)$, where $v_{\text {Disc }}$ is the current discounted valuation of a good that will be delivered in the future, $v_{\text {Nom }}$ is the nominal value of the good- the value that it would have if delivered immediately, and $f(d)$ is some function of $d$, the delay interval. Classic instances of $f(d)$ are exponential, hyperbolic, and hyperbolic-power functions of time, all seen in this journal issue.

This preference for a multiplicative form undoubtedly stems from the interests rates charged or bequeathed by banks, which must provide some measure of scale invariance: The proportional discount, $P_{\text {Disc }}=v_{\text {Disc }} / v_{\text {Nom }}$ must be independent of the dollar value of the deferred good. If for some fixed period of time $P_{\text {Disc }}=0.5$, then $v_{\text {Disc }}(100)=50 ; v_{\text {Disc }}(1000)=500$, and so on. There is no magnitude effect: The discount factor is $f(d)$, not $f\left(d, v_{\text {Nom }}\right)$. If there were a magnitude effect - say that large $v_{\text {Nom }}$ were discounted at lower rates than smaller ones - then an individual or association could make a profit by consolidating loans and receiving a better rate from the bank, part of which savings the financier would pocket. In like manner, if banks did not use the exponential discount function, it would again be possible to make money-pumps out of them. In this case, the financier would manage debts in time, rather than amount. If, for instance, banks used hyperbolic discounting, the financier would profit by marketing slowly discounted long-term debt against highly discounted short-term debt. Although such options are common in the stock market where values fluctuate moment-to-moment, banks must guarantee a fixed return on investment. The only discount function whose rate is independent of time is the exponential function. Market forces would quickly drive hyperbolic bank rates toward the exponential.

Another historic influence on the choice of multiplicative functions has been the economists' and game theorists' models of expected value for probabilistic discounting (e.g., 
Tversky \& Kahneman, 1992; Von Neumann \& Morgenstern, 2007). The use of a form analogous to the standard one for delay discounting (viz., $\left.v_{\text {Disc }}=v_{\text {Nom }} f(p)\right)$ persists despite the many paradoxes that arise from such computations of expected value and expected utility (Allais, 1979; Harless \& Camerer, 1994; Schoemaker, 1982; Segal, 1987).

The constraints on brain and behavior are not the same as the constraints on banks. The evolutions of all are particular to their niches. It therefore makes some sense to liberalize our intuitions, letting the nature of the models of human behavior diverge from those normative for banks and economic theory. Whereas there are infinite numbers of ways we may posit more complex models for human behavior (see, e.g., Doyle, 2013), there are only a few ways that they might be simpler. In particular, consider the hypothesis that humans do not multiply the value of a good by a function of its psychological delay. Instead, humans form a notion of the utility of a good, and they form a notion of the disutility of waiting a certain length of time for it, and simply add those two.

On the one hand this may seem like a stupid suggestion, as the dimensional units do not seem to be the same, and numbers could go negative, and isn't it like adding apples and oranges? It couldn't work. On the other hand, have you ever noticed how increasing the jackpot payoff on lotteries increases the number of purchasers far beyond the lotteries' expected value? This could not happen if the gamblers were rational and computed expected value. We shake our heads at their innumeracy; but perhaps it is our models of rationality that we should be shaking our heads at. If humans operated according to an additive function on probability and amount, the huge utility of the megamillions they might win could easily overwhelm the additive disutility of the small probability of getting it. Pascal's middle-aged wager (Pascal's Wager, 2014) — to give up the fast life because, if there is even a snowball's chance in hell of going to heaven, then heaven's infinite value was worth the gamble_-might make some sense if he was adding utilities; but much less if computing expected value. To infer that gamblers are benighted because they do not adhere to our standards of rational man, or that because one class of people has steeper discount functions than another, that they are impulsive-short-changes our opportunity for understanding what they value and how they make trade-offs. Gamblers 
knowingly pay to play; and sometimes smaller-sooner really is better; just think back to when you weren't middle-aged.

\section{Doing the numbers}

How to make this work? To add numbers, they must have the same dimensions. It is a long tradition in psychophysics to recognize that animals process stimuli in ways that are typically non-linear functions of their magnitude. Doubling the energy of a light or intensity of a tone does not double their brightness or loudness. Power transformations provide a standard and flexible scheme for recoding stimulus magnitude. Let us see how this works for the utility of money.

Twice as much of a thing is seldom twice as good. Certainly it could be the case that twice as much money as you have in your pocket might be just what you need to get in to the show, and then it might be more than twice as good. But twice as many bananas as you bought today would just go brown, twice as much sugar would make your coffee too sweet, and twice as much supper would just make you sick. Twice as much income would be very good indeed; but would it be twice as good? Would it make you exactly twice as happy? The curve that relates delight to dollars is called a utility function.

The utility of a good (a commodity) such as the types used in delay-discounting studies typically have the property called decreasing marginal utility. Twice as much of the thing makes you somewhat less than twice as happy. Here marginal means the derivative, the slopes of the curves in Figure 1, where the utility - think goodness - of a commodity is plotted on the $y$-axis, and its nominal value on the $x$-axis. The slopes of the two curves (their derivatives) decrease with increasing values of the abscissae. In this paper I refer to the $x$-axis as value, and measure it in terms of dollar-value. The name of the units for utilities is utiles, which through happenstance rhymes with smiles. The straight line has a slope of $1 / 2$ : the rate of change in $U(x)$ as a function of $x$ is simply $k=1 / 2$. It is constant for all values of $x$ and thus exemplifies constant marginal utility. The first curved function below it is $U(x)=\sqrt{ } k x$. The margin- the derivative of utility as a function of $x$-is $1 / 2 \sqrt{ }(k / x)$. The marginal utility is decreasing: The slope gets flatter as a function 
of $x$, decreasing proportionately less as $x$ gets larger (as the inverse square root of $x$ in this case). The bottom function is logarithmic: $U(x)=\ln (k x)$. Its derivative is $1 / x$, so more of a thing gets better at an even slower rate.

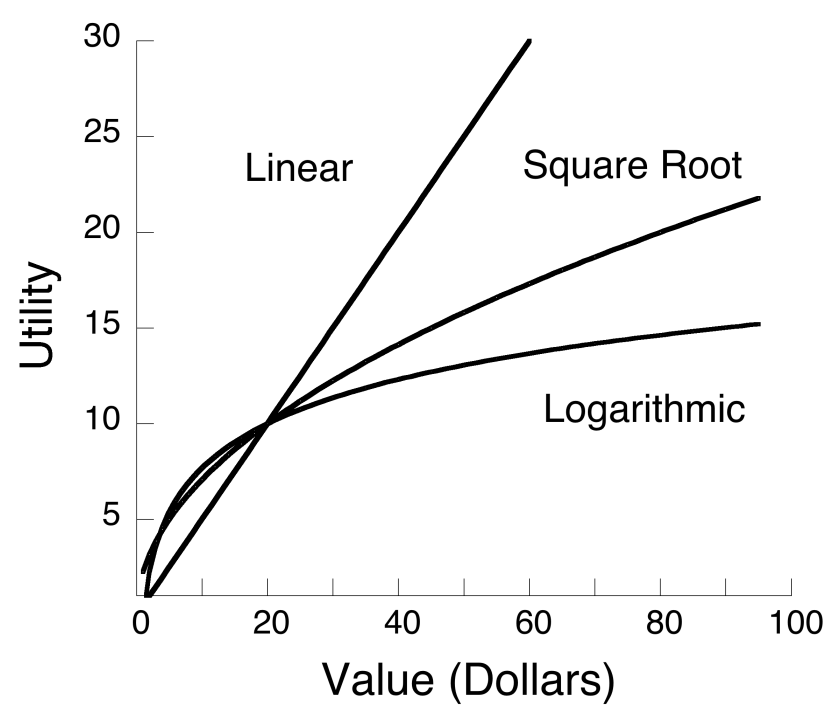

Figure 1. Exemplary utility functions for money.

The linear utility function is the one implicitly assumed in the vast majority of delay discounting studies, which discount dollar value. The second was proposed almost 300 years ago by Cramers as the utility function for money (Pulskamp, 2013). It is consistent with the idea that the utility of new money increases as an inverse function of the utility of the money that we already have. The logarithmic function is consistent with Bernoulli's supposition that utility grows in inverse proportion to the amount of money that we already have. It is the most extreme marginal discounting that we are likely to encounter, so forms the lower bound on plausible utility functions. Power functions converge on the logarithmic function as the power approaches 0 .

We can represent these functions in general as $U(v)=\left(k_{v} v\right)^{\alpha}$. The power $\alpha$ (alpha) is 1 in the case of linear discounting, $1 / 2$ in the case of Cramer discounting, and approaches zero in the case of logarithmic discounting. The coefficient $k_{v}$ is necessary because value may be measured 
in many units - cents, dollars, Euros, pounds - but the utility of four quarters should not be very different from the utility of one dollar bill. This coefficient $k_{v}$ has units of per $\notin$ or per $\$$, etc.

The functions shown in Figure 1 are not just hypothetical. Using magnitude estimation techniques, Galanter (1962) found the power of the utility function for money to be around 0.4. Harinck and associates (Harinck, Van Dijk, Van Beest, \& Mersmann, 2007) used category scaling to estimate the happiness resulting from finding money, and their data were consistent with a power function having an exponent of 0.3 . Thus, the assumption underlying traditional delay discounting tasks is empirically false. What of the assumption underlying the perception of time (delay) in these tasks?

A similar transformation on time is necessary, $U(t)=\left(k_{t} t\right)^{\beta}$. The estimation of elapsed time over small intervals is a power function with exponent just under 1 (Allan, 1983; Eisler, 1976). But when dealing with estimates of future-time over much longer intervals, the exponent shrinks. The difference between today and a week from now is psychologically larger than the difference between 40 and 41 weeks from now. In an experiment in the context of delay discounting, Zauberman and associates (Zauberman, Kim, Malkoc, \& Bettman, 2009) had subjects scale the perceived magnitude of future times, and reported the data shown in Figure 2.

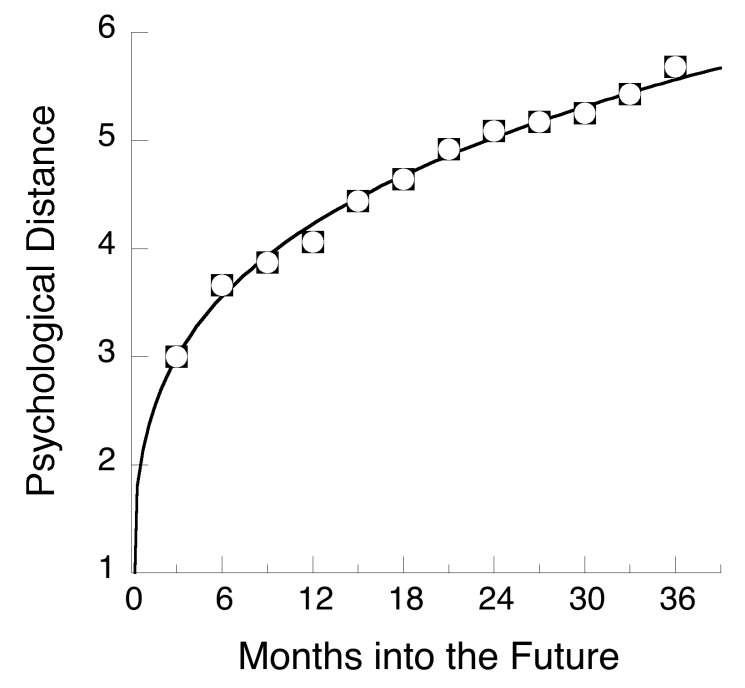

Figure 2. Magnitude estimates of psychological distance to future date. Data from Zauberman eand associates (2009). The curve is a power function with exponent 0.25. 
Adding it all up. According to our additive utility hypothesis, the utility of a good with a nominal value of $v$ delivered at time $t, U(v, t)$, is the weighted combination of these functions on amount and delay.

$$
U(v, t)=w\left(k_{v} v\right)^{\alpha}-(1-w)\left(k_{t} t\right)^{\beta}
$$

The parameter $w$ is the weight that the individual places on the utility of the good, in comparison to the disutility of waiting $(0<w<1)$. Some people may be more patient, or more focused on the value of the good (large $w$ ); others may be on a tighter time schedule or place lower value on the good (small $w$ ). Because a delay in receiving a good is a disutility, the second term in Equation 1 subtracts from the utility of the good. Consider two instances of Equation 1. In the first the value is called $v_{\text {Nom }}$, delivered at a delay of $t=d$; in the second it is called $v_{\text {Disc }}$ and is delivered at a delay of $t=0$ :

$$
\begin{aligned}
& U\left(v_{\text {Nom }}, d\right)=w\left(k_{v} v_{\text {Nom }}\right)^{\alpha}-(1-w)\left(k_{t} d\right)^{\beta} \\
& U\left(v_{\text {Disc }}, 0\right)=w\left(k_{v} v_{\text {Disc }}\right)^{\alpha}
\end{aligned}
$$

The adjusting-amount procedure varies the value of $v_{\text {Disc }}$ until the subject is indifferent between these two utilities. (It is also possible to adjust the delay to the deferred good with a fixed value for the immediate good (e.g., Green, Myerson, Shah, Estle, \& Holt, 2007); those models are left as an exercise for the student.) We may model this indifference as equality between the two utilities:

$$
U\left(v_{\text {Disc }}, 0\right)=U\left(v_{\text {Nom }}, d\right)
$$

substituting,

$$
w\left(k_{v} v_{\text {Disc }}\right)^{\alpha}=w\left(k_{v} v_{\text {Nom }}\right)^{\alpha}-(1-w)\left(k_{t} d\right)^{\beta}
$$

On the right is the current utility of the deferred good whose street value is $v_{\text {Nom. }}$. On the left is the utility of the good delivered immediately, whose value $v_{\text {Disc }}$ has been adjusted until its 
utility equals that of the deferred good. It is dollar value, however, not utilities that are measured in all experiments. To compute what those must be, divide each side by the coefficients $w k_{\mathrm{v}}{ }^{\alpha}$ :

$$
v_{\text {Disc }}^{\alpha}=v_{\text {Nom }}^{\alpha}-k d^{\beta}
$$

The coefficient $k$ compacts a combination of the coefficients from Equation 1:

$$
k=\frac{(1-w)}{w} \frac{k_{t}^{\beta}}{k_{v}^{\alpha}}
$$

This is convenient, as estimation of the constituent parameters would be difficult. Equation 3a makes clear that the rate of discounting the utility of a delayed good, $k$, represents the relative weight on time compared to that on the good in question, along with some arbitrary scale factors associated with the units in which the variables are represented. In the case that the right-hand side of Equation 2 is negative, there is no immediate amount that is small enough, and the offer is rejected.

The original derivation of this theory included the powers as coefficients of $k$ (Equation 3b). It remains an empirical question which version will provide the most parsimonious account of individual differences by rendering the parameters more orthogonal. (I'm betting on $3 \mathrm{~b}$ ).

$$
k=\frac{(1-w)}{w} \frac{k_{t}^{\beta}}{k_{v}^{\alpha}} \frac{\alpha}{\beta}
$$

Finally, to deliver the prediction in terms of the currency of the experiment, raise each side of Equation 2 to the power $1 / \alpha$ (keeping $\alpha>0)$ :

$$
v_{\text {Disc }}=\left(v_{\text {Nom }}^{\alpha}-k d^{\beta}\right)^{1 / \alpha}
$$

This is the key equation of the additive utility model. In the case that the disutility of waiting exceeds the utility of the nominal amount offered, $k d^{\beta}>v_{\text {Nom }}^{\alpha}$, then $v_{\text {Disc }}=0$.

As the value of $\alpha$ approaches 0 , the power-utility function for the good may be represented by a power series (see the appendix). In that case, and invoking Equation $3 \mathrm{~b}$ for the expansion of $k$, Equation 4 may be written as: 


$$
v_{\text {Disc }}=v_{\text {Nom }} e^{-k^{\prime} d^{\beta}}
$$

The series expansion of this exponential (see the appendix) is the familiar:

$$
v_{D i s c}=\frac{v_{N o m}}{1+k^{\prime} d^{\beta}}
$$

This traditional form is thus consistent with a very concave or logarithmic utility function for money and its additive combination with the disutility of delay. Because the utility of the good does not enter Equations 5 and 6, they are useful whenever value is not independently varied along with delay, giving the same results as Equation 4 (with changes in the parameter values), but saving the now redundant parameter $\alpha$. The rates of discounting in Equations 5 and

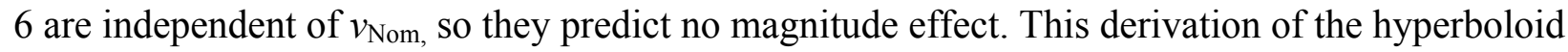
predicts that the rate of decay $k$ ' will be inversely proportional to the power $\beta$ (see the appendix).

Equation 5 is interesting because it returns the bankers' discount function, corrected for nonlinearity in the future-time function. It has been suggested as a discounting model by Ebert and Prelec (2007). It restores bankers' rationality to discounting decisions if we assume a steeply curved utility function for money $\alpha \rightarrow 0$, and a nonlinear valuation of future-time. Their article is interesting because it demonstrates how the temporal discount function (in particular, the value of $\beta$ ) is readily subject to manipulation. We may infer that some of the large heterogeneity in discounting that is found in typical studies may be due to idiosyncratically perturbed future perspectives of the subjects (term papers or rents imminent in one case, an endless summer lying ahead in another). If so, standard framing instructions may help control this unwonted variability. Alternatively, using existing techniques (see, e.g., Zauberman et al., 2009), future perspective might be measured and used as a covariate.

According to the additive discount model of Equation 4, the apparent rate of discounting will depend on the magnitude of the value that is discounted. Imagine discounting a good of large value where $v_{\text {Nom }}^{\alpha}>>d^{\beta}$. Then the additive temporal disutility would be relatively negligible, and discounted value would essentially equal the nominal value. The utility of heaven is essentially the same whether it comes in days or in decades. For the same reason the state of 
the planet 100 years from now is so distant that no economic models with sensible exponential discount rates would counsel expending many resources abating climate change today (Dasgupta, 2006). But there are some individuals for whom the utility of a viable planet for their children's children is so great that reductions in carbon emission are of focal importance despite their deferred payoff. This makes sense in the additive model, without invoking the issue of intergenerational equity (Portney \& Weyant, 1999). Conversely, for goods of little value time becomes of the essence, with any delay deadly to the enterprise.

\section{The problem of scale invariance}

All standard discounting models of the form $v_{\text {Disc }}=v_{\text {Nom }} f(d)$ are scale invariant over the nominal value: They must predict discounting at the same rate for all values. When the delay $v_{\text {Disc }}$ is 0 , then the proportional value of the nominal good as a function of delay is $P_{\text {Disc }}=v_{\text {Disc }} / v_{\text {Nom }}=f(d)$, for all $v_{\text {Nom. }}$. This is also true of Equations 5 and 6 . This is counter to the facts of human delay discounting, so all such models must deal with that invalidation by ad hoc adjustments of the discount rate $k$. Equation 4 shows how a model with parameter invariance can predict scale variance.

Figure 3 shows representative predictions of the model with $\alpha=0.09, \beta=0.65$, and $k=$ 0.007 for time measured in months. Overlaying the curves are classic data from Green and associates (Green, Myerson, \& McFadden, 1997). Note that at 20 years $P_{\text {Disc }} \approx 0.2$ for $\$ 100$ and $\approx 0.4$ for $\$ 100 \mathrm{~K}$. The failure of scale invariance becomes more obvious than that visible in Figure 3 when delay discount experiments are done in real-time. Imagine being in a real-time delay-discount experiment where you must wait 20 minutes for a unit payoff. What will you take immediately rather than that delayed amount? In all likelihood you would discount it steeply, especially if it is small, and the discount function would plummet invisibly close to one of the $y$ axes in Figure 3 (see Johnson, Hermann, \& Johnson, 2014). The disutility of waiting depends on what is bundled with it: If it is life as usual, the weight on time will be small; if it is solitary confinement in a laboratory, it will be large (Paglieri, 2013). 


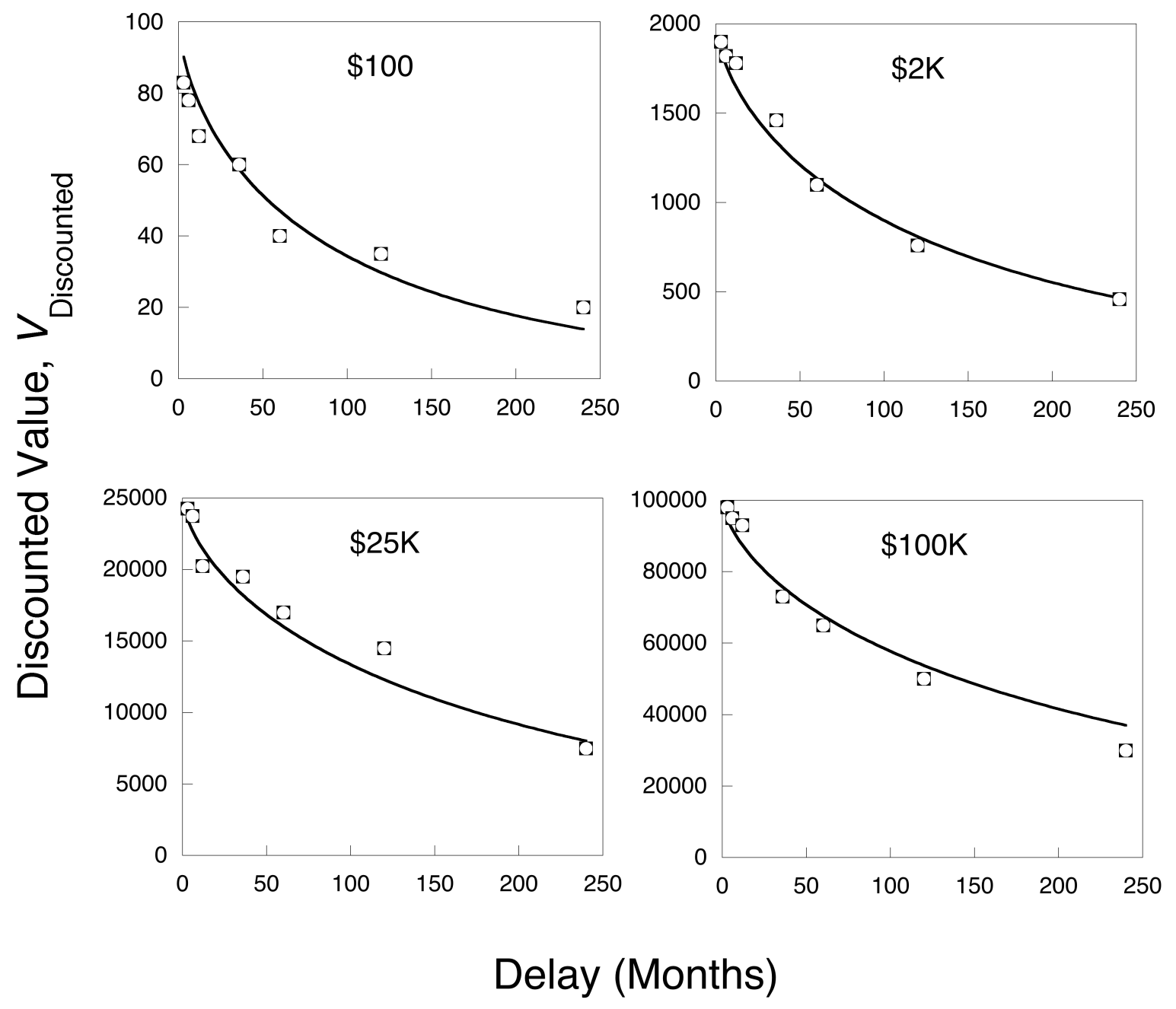

Figure 3. The time course of Equation 2 as a function of magnitude and delay of a hypothetical payoff. Despite the apparently steeper discounting of small amounts, the three key parameters of Equation 2 were the same across all panels. Data from (Green, Myerson, \& McFadden, 1997).

\section{Disutilities}

What if we are threatened with a loss, rather than promised a gain? The arithmetic should continue to work if we couch the problems correctly. (Such couching is the creative part of doing this kind of arithmetic, as it involves modeling how the subjects frame the losses and gains. Tversky \& Kahneman, 1981). Suppose that you are offered the ability to pay to decrease the cost 
of a future assessment of 200 dollars. The simplest framing of this decision is to ask for what value $x$ the disutility of paying it now would just balance the disutility of paying $\$ 200$ after a delay of $d$ months. Of course, you must also assume that you have the money in your account to be able to pay - call that $v_{\text {Acct. }}$ This situation recasts Equation 2 as $v_{A c c t}^{\alpha}-v_{D i s c}^{\alpha}=v_{A c c t}^{\alpha}-v_{N o m}^{\alpha}+k d^{\beta}$. Now the utility of the delay is positive, as we are willing to pay to delay: witness credit cards. After rearranging, this framing leads to Equation 2 and thence 4. Figure 4 shows the model aligned with data from the third experiment of Estle, Green, Myerson, and Holt (2006). They show a large magnitude effect for gains, reflected in a relatively large value for $\alpha$, and a smaller one for losses (the magnitude effect was not found to be significant for losses in the analysis of parameters from individual data). Losses showed a smaller rate of discounting $(k)$ than wins. Perhaps a better framing of the arithmetic will reveal an invariance lurking behind this apparent difference; or perhaps different $\alpha$ 's and $k$ 's for wins and losses tells us something distinctive and irreducible about the utility of gains and losses.

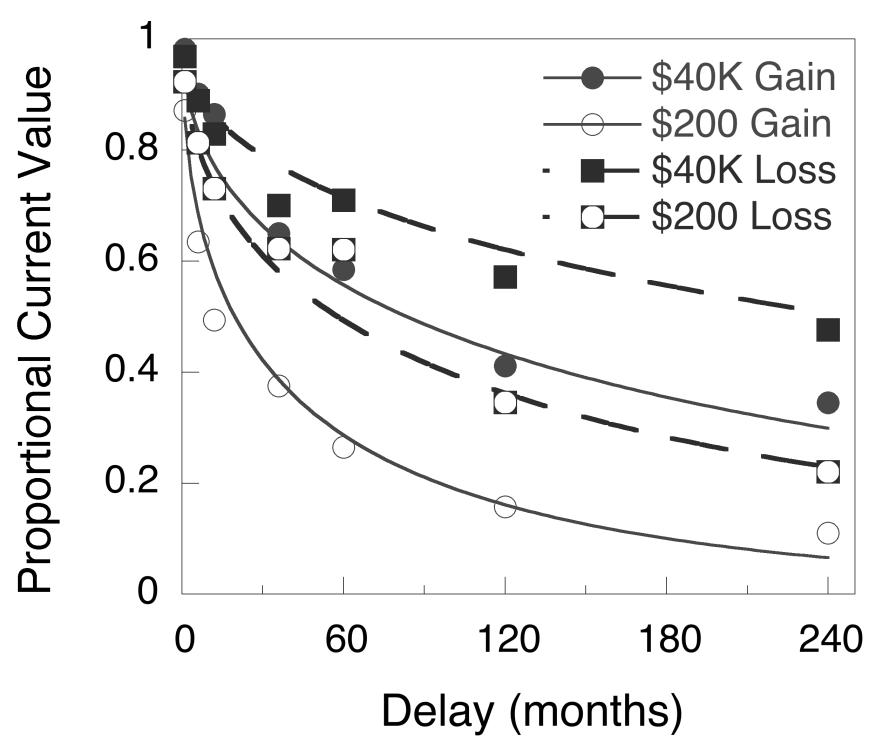

Figure 4. The discount functions for gains and losses, from Estle et al. (2006). The circles depict gains of $\$ 40,000$ (filled) or $\$ 200$ (unfilled). The squares depict large and small losses, following the same convention. The curves are from Equation 2 (divided by the magnitude of loss or win), with $\alpha=0.135, \beta=0.49$, and $k=0.42$ for gains and 0.25 for losses. 
A very convincing absence of magnitude effects was found in a recent study (Green, Myerson, Oliveira, \& Chang, 2014) for subjects choosing between immediate and deferred payments (losses) over a large range of amounts. Why should there be no magnitude effect for payments in general, if that is the case - as other studies (e.g., Mitchell \& Wilson, 2010) also find? One possibility is that the (dis)utility function for losses really has a near-zero power $\alpha$, corresponding to the lowest curve in Figure 1. Once a person has incurred some debt, additional debt is just another drop in the bucket — in for a penny, in for a pound — and best not to think too hard on it. Perhaps the debt becomes in some sense imaginary once it exceeds the current $v_{\text {Acct }}$. Why was there then a (small) magnitude effect for losses in two experiments in the former (Estle et al., 2006) study? Possibly in the $50 \%$ of the cases when the receipts conditions preceded the payments condition, that primed the subjects to be more sensitive to magnitudes.

When the data suffice to support 3 parameters, the additive utility model provides more useful information than some other models. It tells us how nonlinearities in psychological time and in the utility of goods may interact to determine choice. It prevents us from confusing different discount rates with differences in time horizons or in marginal utilities of the deferred good. Figure 5 shows how this might happen (note the logarithmic $x$-axis). Because the utility function for money is straighter (although still quiet curved), the additional amounts available by waiting are worth waiting for. But in the case of the very curved utility function for food, a large number of additional bananas or pizzas or pudding will add very little additional utility. Thus the food curve drops quickly_ not because the discount rate is higher (it is not: $k$ is the same for all curves) - but because the subjects are not greedy for amounts that add very little quality to their lives (as reflected in the different values of $\alpha$ ). 


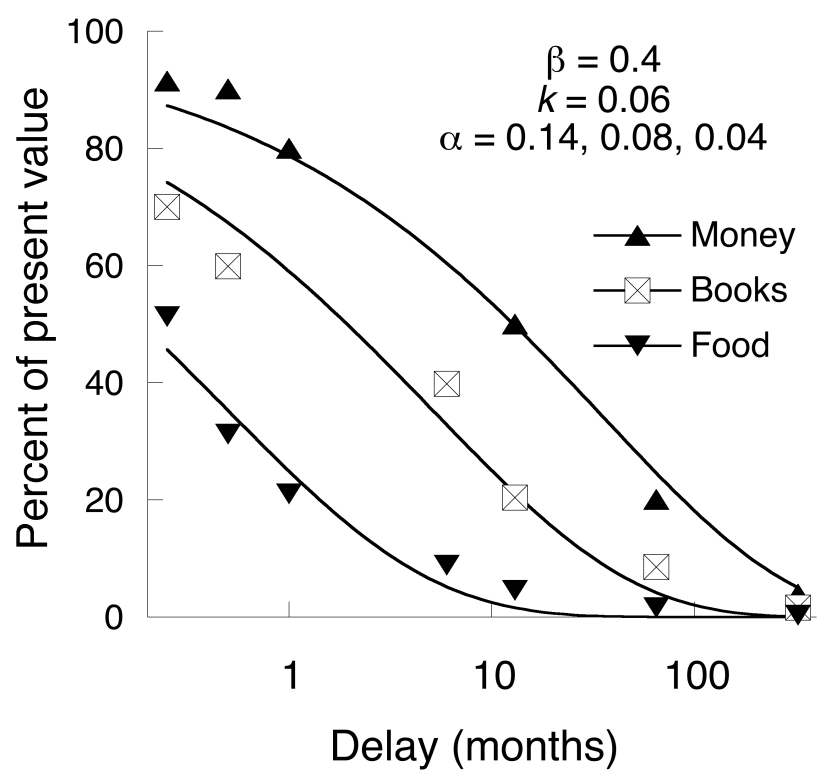

Fig. 5. The discount functions for three commodities, from Charlton and Fantino (2008). The $x$ axis has been logarithmically transformed to increase clarity of presentation. Equation 4 drew the lines, with only the utility function for goods varying. Food satiated most quickly $(\alpha=0.04)$, followed by books, and money was slowest to satiate $(\alpha=0.14)$.

\section{Rat economics}

Animals less verbal than humans deal poorly with hypothetical stipulations of delays to amounts of reinforcement. They must be conditioned to choose between stimuli associated with particular amounts and delays. The simplest exponential delay of reinforcement gradient, averaged over the duration of the stimulus, estimates its conditioned strength:

$$
s(v, d) \propto u(v) \frac{1-e^{-k d}}{k d}
$$

In deriving Equation 7, the area under the exponential trace from the onset of the stimulus until the delivery of the reinforcer is summed (the numerator of Equation 7 over $k$ ) and divided by its duration $d$ to compute its average strength. The exponential form of the gradient is based 
on some evidence and argument (e.g., Johansen, Killeen, Russell, et al., 2009; Johansen, Killeen, $\&$ Sagvolden, 2009). In the limit as $d \rightarrow 0, s(v, d) \rightarrow u(v)$.

The conditioned reinforcing strength, $s(v, d)$, is proportional to the strengthening effect of that amount of reinforcement, $u(v)$, delivered at that delay. The function $u(v)$ is typically concave (e.g., Killeen, 1985): 16 pellets are less than twice as reinforcing as 8 pellets. Equation 7 is a multiplicative, not additive model, however, therefore no magnitude effect is predicted-or found (Oliveira, Green, \& Myerson, 2014). The discount parameter $k$ is a measure of the steepness of the delay of reinforcement gradient. The curves drawn by Equation 7 are indistinguishable from those drawn by Equation 6 (Killeen, 2011, Eqs. 2 \& 3). Equation 7 places all the emphasis on the conditioned reinforcement strength of the stimulus signaling the options, but for nuance, the role of direct reinforcement of the choice response by the delayed primary reinforcer may be taken into account (Killeen, 2011).

\section{Preference}

Some experiments use a different paradigm to assay delay discounting: They measure the proportion of responses for one good over another without adjusting to indifference. This yields a very different dependent variable. There is no reason that degree of preference should follow a hyperbolic function, or even a relative measure of strength (despite some misguided suggestions that it should, e.g., Killeen, 2011). If I repeatedly gave you the choice between $\$ 100$ and \$200 I would be quite surprised if you chose the larger amount only $2 / 3$ of the time. If I repeatedly gave you the choice between $\$ 100$ and $\$ 120$ I would be at least as surprised if you chose the larger amount only $55 \%$ of the time. Animals should always choose what they most prefer, assuming that they can make that judgment. But concurrent reinforcement schedules, in which degree of preference is routinely measured, is a confusing situation. Entering such a situation, clear and near-exclusive preferences are soon degraded (Crowley \& Donahoe, 2004). How to apply the arithmetic of discounting here? It is worth assaying a classic off-the-shelf model of confusion, Thurstone scaling (Thurstone, 1927) incorporated into signal detection theory as its foundational 
model. Other models of schedule confusion are available (e.g., Nevin, 1981) and worth exploring.

Thurstone's confusion model represents the utility of packages of goods and delays not as exact values, but as distributions around a mean. Adjustment procedures titrate some parameter so that the distributions of utilities of two packages of goods-plus-delays align at a common mean. Preference procedures leave them as two distributions on a line of utility, partially overlapping. The probability that one package, say $s\left(v_{1}, d_{1}\right)$, will seem better than another, say, $s\left(v_{2}, d_{2}\right)$, at the moment of choice is most concisely given by a cumulative normal distribution:

$$
p\left(O_{1}\right)=\Phi\left(s\left(v_{1}, d_{1}\right)-s\left(v_{2}, d_{2}\right)-b, \sigma\right)
$$

The probability of choosing Option 1 is a cumulative normal function $(\Phi)$ of the difference between the two strengths, less a bias parameter $b$, with a standard deviation of $\sigma$ (sigma). When sigma is very small, the function is a step-function, the subject always preferring the better (say, the large delayed option) until a parameter (say, its delay) becomes sufficiently large that it prefers the alternative option. As $\sigma$ gets larger, the function flattens into an ogive. A logistic distribution would work as well as the normal here. Figure 6 shows an exemplary application of Equation 8, employing Equation 7 to measure strength, and keeping $b=0$. Although both parameters drove the SHR rats toward a steeper function, it is possible to construct steep functions with either of the parameters. Equation 8 may also be used for human preferences, substituting $U\left(v_{i}, d_{i}\right)$ for $s\left(v_{i}, d_{i}\right)$. A version of the Thurstone model has been used to study memory (White \& Wixted, 1999). Davison and Nevin (1999) have used a similar logistic model to develop a general theory of the relations among responses stimuli and reinforcers. 


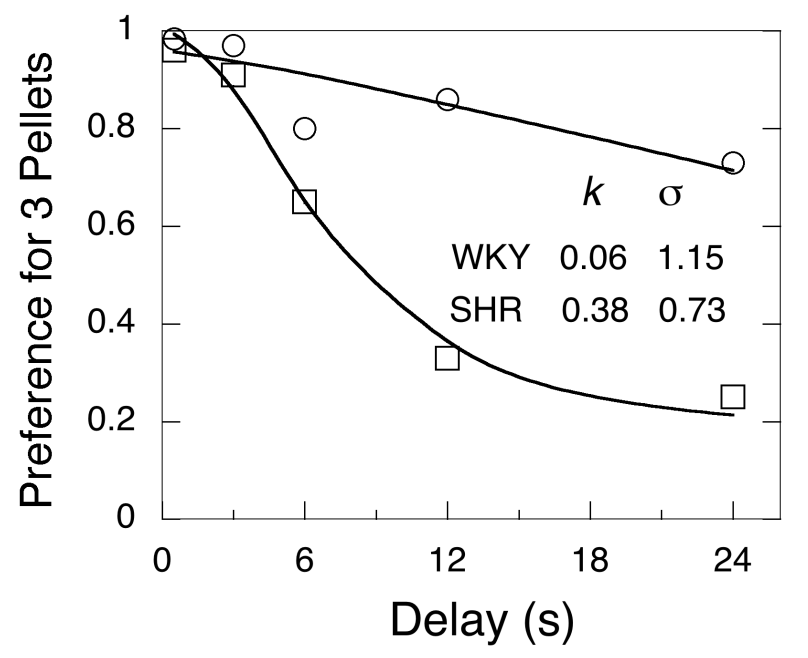

Fig. 6. Data from Fox, Hand, and Reilly (2008), who offered two strains of rats (WKY and SHR) the choice of 1 pellet delivered immediately or 3 after the delay indicated on the $x$-axis.

In deploying Equation 7 for only two amounts, it sufficed to set $u(1)=1$ and $u(3)=3$, but this will not always be the case. Because these measures of reinforcing strength do not cancel when differences (rather than ratios) are taken, there will be a kind of magnitude effect found with this model. Doubling both amounts will move the distributions apart (akin to reducing $\sigma$ ) so that preferences on either side of the indifference point will get more extreme. It is notable that all of the reports of magnitude effects (or reverse magnitude effects) with rats used the preference paradigm (Oliveira et al., 2014, Table 3), whereas there was no evidence of magnitude effects in non-verbal animals using the adjustment paradigm.

\section{Discussion}

Arithmetic is relatively easy, but deciding what to add requires a sense of how the organism evaluates the options available to it, and how those might be affected by the framing of the experimental paradigm. It requires experimentation with the math, as much as with the rats. Therefore all of the above models should be viewed as hypotheses. The readers are invited to do their own sums. In addition they might test some of the qualitative predictions of this simple but strange approach, some of which are found in (Killeen, 2009). Serious evaluation of the models requires analyses of data from individual subjects, as averaging curvilinear functions can mislead. Green, Myerson, and their collaborators provide many examples of the proper ways to analyze such data. 


\section{References}

Allais, M. (1979). The so-called Allais paradox and rational decisions under uncertainty Expected utility hypotheses and the Allais paradox (pp. 437-681): Springer.

Allan, L. G. (1983). Magnitude estimation of temporal intervals. Perception and Psychophysics, 33, 29-42. doi: http://dx.doi.org/10.3758/BF03205863

Burington, R. S. (1948). Handbook of mathematical tables and formulas. Sandusky, OH: Handbook Publishers, Inc.

Charlton, S. R., \& Fantino, E. (2008). Commodity specific rates of temporal discounting: does metabolic function underlie differences in rates of discounting? Behavioural Processes, 77(3), 334-342. doi: http://dx.doi.org/10.1016/j.beproc.2007.08.002

Crowley, M. A., \& Donahoe, J. W. (2004). Matching: its acquisition and generalization. Journal of the Experimental Analysis of Behavior, 82(2), 143-159. doi: http://dx.doi.org/10.1901/jeab.2004.82-143

Dasgupta, P. (2006). Comments on the Stern Review's economics of climate change. Foundation for Science and Technology, http://econ.tau.ac.il/papers/research/Partha Dasgupta on Stern Review.pdf.

Davison, M., \& Nevin, J. A. (1999). Stimuli, reinforcers and behavior: An integration. Journal of the Experimental Analysis of Behavior, 71, 439-482. doi: 10.1901/jeab.1999.71-439

Doyle, J. R. (2013). Survey of time preference, delay discounting models. Judgment and Decision Making, 8(2), 116-135. doi: http://dx.doi.org/10.2139/ssrn.1685861

Ebert, J., \& Prelec, D. (2007). The fragility of time: Time-insensitivity and valuation of the near and far future. Management Science, 53, 1423-1438. doi: http://dx.doi.org/10.1287/mnsc.1060.0671

Eisler, H. (1976). Experiments on subjective duration 1868-1975: A collection of power function exponents. Psychological Bulletin, 83(6), 1154. doi: http://dx.doi.org/10.1037/00332909.83.6.1154

Estle, S. J., Green, L., Myerson, J., \& Holt, D. D. (2006). Differential effects of amount on temporal and probability discounting of gains and losses. Memory and Cognition, 34, 914-928. doi: http://dx.doi.org/10.3758/BF03193437 
Fox, A. T., Hand, D. J., \& Reilly, M. P. (2008). Impulsive choice in a rodent model of attentiondeficit/hyperactivity disorder. Behavioural Brain Research, 187(1), 146-152. doi: http://dx.doi.org/10.1016/j.bbr.2007.09.008

Galanter, E. (1962). The direct measurement of utility and subjective probability. American Journal of Psychology, 72, 208-220. doi: http://dx.doi.org/10.2307/1419604

Green, L., Myerson, J., \& McFadden, E. (1997). Rate of temporal discounting decreases with amount of reward. Memory and Cognition, 25, 715-723. doi: http://dx.doi.org/10.3758/BF03211314

Green, L., Myerson, J., Oliveira, L., \& Chang, S. E. (2014). Discounting of delayed and probabilistic losses over a wide range of amounts. Journal of the Experimental Analysis of Behavior, 101(2), 186-200. doi: http://dx.doi.org/10.1002/jeab.56

Green, L., Myerson, J., Shah, A. K., Estle, S. J., \& Holt, D. D. (2007). Do adjusting-amount and adjusting-delay procedures produce equivalent estimates of subjective value in pigeons? Journal of the Experimental Analysis of Behavior, 87(3), 337-347. doi: http://dx.doi.org/10.1901/jeab.2007.37-06

Harinck, F., Van Dijk, E., Van Beest, I., \& Mersmann, P. (2007). When gains loom larger than losses: Loss aversion for small amounts of money. Psychological Science, 18, 10991105. doi: http://dx.doi.org/10.1111/j.1467-9280.2007.02031.x

Harless, D. W., \& Camerer, C. F. (1994). The predictive utility of generalized expected utility theories. Econometrica: Journal of the Econometric Society, 1251-1289. doi: http://dx.doi.org/10.2307/2951749

Johansen, E. B., Killeen, P. R., Russell, V. A., Tripp, G., Wickens, J. R., Tannock, R., . . . Sagvolden, T. (2009). Origins of altered reinforcement effects in ADHD. Behavioral and Brain Functions, 5, 7. doi: http://dx.doi.org/10.1186/1744-9081-5-7

Johansen, E. B., Killeen, P. R., \& Sagvolden, T. (2009). Behavioral variability, elimination of responses, and delay-of-reinforcement gradients in SHR and WKY rats. Behavioral and Brain Functions, 3. doi: http://dx.doi.org/10.1186/1744-9081-3-60

Johnson, P. S., Herrmann, E. S., \& Johnson, M. W. (2014). Opportunity costs of reward delays and the discounting of hypotheical money and cigarettes. Journal of the Experimental Analysis of Behavior. Advance online publication. DOI: 10.1002/jeab.110 
Killeen, P. R. (1985). Incentive theory IV: Magnitude of reward. Journal of the Experimental Analysis of Behavior, 43, 407-417. doi: http://dx.doi.org/10.1901/jeab.1985.43-407

Killeen, P. R. (2009). An additive-utility model of delay discounting. Psychological Review, 116, 602-619. doi: http://dx.doi.org/10.1037/a0016414

Killeen, P. R. (2011). Models of trace decay, eligibility for reinforcement, and delay of reinforcement gradients, from exponential to hyperboloid. Behavioural Processes, 8(1), 57-63. doi: http://dx.doi.org/10.1016/j.beproc.2010.12.016

Mitchell, S. H., \& Wilson, V. B. (2010). The subjective value of delayed and probabilistic outcomes: Outcome size matters for gains but not for losses. Behavioural Processes, 83(1), 36-40. doi: http://dx.doi.org/10.1016/j.beproc.2009.09.003

Nevin, J. A. (1981). Psychophysics and reinforcement schedules: An integration. In M. L. Commons \& J. A. Nevin (Eds.), Quantitative Analysis of Behavior: Discriminative Prperties of Reinforcement Schedules (Vol. 1, pp. 3-27). Cambridge, MA: Ballinger.

Oliveira, L., Green, L., \& Myerson, J. (2014). Pigeons' delay discounting functions established using a concurrent-chains procedure. Journal of the Experimental Analysis of Behavior, 102(2), 151-161. doi: http://dx.doi.org/10.1002/jeab.97

Paglieri, F. (2013). The costs of delay: Waiting versus postponing in intertemporal choice. Journal of the Experimental Analysis of Behavior, 99, 362-377. doi: 10.1002/jeab.18

Pascal's Wager. (2014, Nov. 10). In Wikipedia, The Free Encyclopedia. Retrieved Nov. 20, 2014, from http://en.wikipedia.org/w/index.php?title=Pascal\%27s_Wager\&oldid=633303305

Portney, P. R., \& Weyant, J. P. (Eds.). (1999). Discounting and intergenerational equity. Washington, DC: Resources for the Future.

Pulskamp, R. J. (2013). Correspondence of Nicolas Bernoulli concerning the St. Petersburg Game. 2007, 1-9. cerebro.xu.edu/math/Sources/NBernoulli/correspondence_petersburg_game.pdf

Schoemaker, P. J. H. (1982). The expected utility model: Its variants, purposes, evidence and limitations. Chicago: Center for Decision Research, The University of Chicago.

Segal, U. (1987). The Ellsberg paradox and risk aversion: An anticipated utility approach. International Economic Review, 175-202. doi: http://dx.doi.org/10.2307/2526866 
Thurstone, L. L. (1927). A law of comparative judgment. Psychological Review, 34, 273-286. doi: http://dx.doi.org/10.1037//0033-295X.101.2.266

Tversky, A., \& Kahneman, D. (1981). The framing of decisions and the psychology of choice. Science, 211, 453-458. doi: http://dx.doi.org/10.1126/science.7455683

Tversky, A., \& Kahneman, D. (1992). Advances in prospect theory: Cumulative representation of uncertainty. Journal of Risk and Uncertainty, 5(4), 297-323. doi: http://dx.doi.org/10.1007/BF00122574

Von Neumann, J., \& Morgenstern, O. (2007). Theory of Games and Economic Behavior (60th Anniversary Commemorative Edition). Princeton, NJ: Princeton University Press.

White, K. G., \& Wixted, J. T. (1999). Psychophysics of remembering. Journal of the Experimental Analysis of Behavior, 71(1), 91-113. doi: 10.1901/jeab.1999.71-91

Zauberman, G., Kim, B. K., Malkoc, S. A., \& Bettman, J. R. (2009). Discounting time and time discounting: Subjective time perception and intertemporal preferences. Journal of Marketing Research, 46(4), 543-556. doi: http://dx.doi.org/10.1509/jmkr.46.4.543 


\section{Appendix}

The limit as $\alpha \rightarrow 0$.

Restating Equation 2:

$$
v_{D i s c}^{\alpha}=v_{N o m}^{\alpha}-k d^{\beta}
$$

The Maclaurin series expansion of $v^{\alpha}$ is (Burington, 1948, p. 44):

$$
v^{\alpha}=1+\alpha \ln (v)+\frac{(\alpha \ln (v))^{2}}{2 !}+\ldots
$$

For small values of $\alpha$ all but the first two terms of the series may be ignored, as higher powers of $\alpha$ quickly become minuscule. Substituting those into Equation A1:

$$
1+\alpha \ln \left(v_{D i s c}\right)=1+\alpha \ln \left(v_{\text {Nom }}\right)-k d^{\beta}
$$

Rearranging:

$$
\ln \left(v_{\text {Disc }}\right)=\ln \left(v_{\text {Nom }}\right)-\frac{k}{\alpha} d^{\beta}
$$

Exponentiating:

$$
v_{\text {Disc }}=v_{\text {Nom }} e^{-\frac{k}{\alpha} d^{\beta}}
$$

Here is where taking Equation $3 \mathrm{~b}$ as the proper expansion of $k$ is useful, as the parameter alpha cancels out of the exponent, avoiding division by zero in the limiting case:

$$
v_{D i s c}=v_{N o m} e^{-k^{\prime} d^{\beta}}
$$

with

$$
k^{\prime}=\frac{(1-w)}{w \beta} \frac{k_{t}^{\beta}}{k_{v}^{\alpha}}
$$

\section{Reducing to the hyperboloid}

Equation A6 may be written as: 


$$
v_{D i s c}=\frac{v_{N o m}}{e^{k^{\prime} d^{\beta}}}
$$

Notice that if $v$ in Equation $\mathrm{A} 2$ is $e$, then it may be written as:

$$
e^{\alpha}=1+\alpha+\frac{\alpha^{2}}{2 !}+\ldots
$$

In this case, $\alpha=k^{\prime} d^{\beta}$. Then substituting the first two terms for the denominator of Equation A8, that may be written as:

$$
v_{\text {Disc }}=\frac{v_{\text {Nom }}}{1+k^{\prime} d^{\beta}}
$$

This is the standard hyperboloid discounting function. Note that $k$ ' has $\beta$ in its denominator (Equation A7). This derivation of the hyperboloid therefore predicts that the discount rate will be inversely proportional to the exponent $\beta$. 
